Research Article

\title{
Network Pharmacology-Based Analysis of the Underlying Mechanism of Hyssopus cuspidatus Boriss. for Antiasthma: A Characteristic Medicinal Material in Xinjiang
}

\author{
Rongchang Liu $\mathbb{D},{ }^{1}$ Yan Mao $\mathbb{D},{ }^{2}$ Zhengyi Gu $\mathbb{D},{ }^{2}$ and Jinhua He $\mathbb{D}^{2}$ \\ ${ }^{1}$ College of Pharmacy, Xinjiang Medical University, Urumqi 830011, China \\ ${ }^{2}$ Xinjiang Institute of Materia Medica, Urumqi 830004, China
}

Correspondence should be addressed to Yan Mao; maoyan7529@163.com, Zhengyi Gu; zhengyi087@126.com, and Jinhua He; hejh1216@163.com

Received 28 July 2021; Accepted 26 October 2021; Published 29 November 2021

Academic Editor: Changmin $\mathrm{Hu}$

Copyright $(2021$ Rongchang Liu et al. This is an open access article distributed under the Creative Commons Attribution License, which permits unrestricted use, distribution, and reproduction in any medium, provided the original work is properly cited.

Background. Hyssopus cuspidatus Boriss. (Shen Xiang Cao (SXC)), a traditional medicine herb in Xinjiang, has a long history of being used by minorities to treat asthma. However, its active antiasthmatic compounds and underlying mechanism of action are still unknown. The aim of this study was to investigate the bioactive compounds and explore the molecular mechanism of SCX in the treatment of asthma using network pharmacology. Methods. The compounds of SCX were collected by a literature search, and Traditional Chinese Medicine Systems Pharmacology Database and Analysis Platform (TCMSP) and SwissTargetPrediction were used to predict targets and screen active compounds. Moreover, asthma-related targets were obtained based on DisGeNET, Herb, and GeneCards databases, and a protein-protein interaction (PPI) network was built by the STRING database. Furthermore, the topological analysis of the PPI and SXC-compound-target networks were analyzed and established by Cytoscape software. Finally, the RStudio software package was used for carrying out Gene Ontology (GO) function enrichment and Kyoto Encyclopedia of Genes and Genomes (KEGG) pathway analysis. AutoDock tools and AutoDock Vina were used to molecularly dock the active compounds and key targets. Results. A total of 8 active compounds and 258 potential targets related to SXC were predicted, and PPI network screened out key targets, including IL-6, JUN, TNF, IL10, and CXCL8. GO enrichment analysis involved cell responses to reactive oxygen species, oxidative stress, chemical stress, etc. In addition, KEGG pathway analysis showed that SXC effectively treated asthma through regulation of mitogen-activated protein kinases (MAPK) signaling pathways, interleukin 17 (IL-17) signaling pathways, toll-like receptor (TLR) signaling pathways, and tumor necrosis factor (TNF) signaling pathways. Conclusion. The preliminary study that was based on multiple compounds, multiple targets, and multiple pathways provides a scientific basis for further elucidating the molecules involved and the underlying antiasthma-related mechanisms of SXC.

\section{Introduction}

Asthma is a type of respiratory tract allergic inflammatory disease in which mast cells and airway epithelial cell dysfunction play an important role $[1,2]$. The predominant clinical symptoms of asthma include chest tightness, coughing, wheezing, and shortness of breath [3]. Although corticosteroids are considered as the first-line clinical drug to relieve the symptoms of patients [4], it cannot completely cure, or even aggravate symptoms of asthma sometimes [5]. In recent studies, it has been shown that asthma is likely related to epigenomes and environmental factors $[6,7]$. According to a national cross-sectional study, it was estimated that there are currently about 47.5 million patients with asthma in China and that some patients have not yet been effectively diagnosed and treated [8]. Furthermore, the incidence of asthma in Chinese children aged 0-14 years is gradually increasing [9]. The complex pathogenesis of asthma is still unclear. At present, it is widely believed that the balances of Th1/Th2 and TH17/Treg play an important role in the pathogenesis of asthma. [10]. Th2 cells can release various cytokines, such as IL-4, IL-5, and IL-13. These 
cytokines could drive the recruitment of eosinophil cells to accumulate in the lung [11]. The cytokine, IFN- $\gamma$, produced by Th1 cells can inhibit Th2 cell proliferation, induce eosinophil apoptosis, and antagonize the inflammatory factor [12]. Th17 produces various cytokines including IL-17, IL-6, and TNF- $\alpha$ [13]. TNF- $\alpha$ is a key inflammatory mediator that is produced by macrophages. It is a regulatory factor in the immune response and in cell proliferation and differentiation [14]. IL-6 is a proinflammatory cytokine produced after immune activation that can aggravate chronic inflammation [15].

Traditional medicine in Xinjiang is an important medicinal system of Traditional Chinese Medicine (TCM), and it is one of the part of medicines in China. Shen Xiang Cao (SXC, Labiatae family) comes from the dried ground aerial parts of Hyssopus cuspidatus Boriss. and is mainly distributed in Altay, Tianshan, and Kunlun Mountains [16, 17].

Previous studies performed by Jinhua He's team showed that different elution (water and ethanol) of SXC has a certain antagonistic effect on the acetylcholine-induced or histamine-induced smooth muscle contraction of isolated tracheal in guinea pigs, and $60 \%$ ethanol elution shows the strongest effect, which has similar effects with aminophylline at high mass concentration [18]. Furthermore, an ethanol extract of SXC has an anti-inflammatory effect and inhibits the production of inflammatory-related factors in RAW264.7 cells and activation of the NF- $\kappa$ B signaling pathway and MAPK signaling pathway [19]. Moreover, it was found that SXC has a certain regulatory effect on Th1 and Th2 immune responses as well as a relaxing effect on bronchial smooth muscle, which may have an antagonistic effect on the $\mathrm{M}$ cholinergic receptor and $\mathrm{H} 1$ receptors [20]. As a traditional folk medicine, SXC is frequently used by Xinjiang minorities to treat cough, asthma, and wheezing [21]. However, the basis of the compound and the underlying antiasthma mechanism of action are still unclear. Therefore, the aim of this study was to research the targets and mechanisms of action of the active ingredients of SXC with the overall perspective of network pharmacology to explore the underlying mechanism of SXC in the antiasthma process.

\section{Materials and Methods}

2.1. Collection of Bioactive Compounds and Targets. "Hyssopus cuspidatus Boriss." was used as a key search term in the China National Knowledge Infrastructure (CNKI) database (https://www.cnki.net/), Wanfang database (http:// www.wanfangdata.com.cn/index.html), Weipu database (http://www.cqvip.com/), and PubMed database (https:// pubmed.ncbi.nlm.nih.gov/) to obtain chemical compounds of SXC. Subsequently, TCMSP (https://tcmspw. com/tcmsp.php) were adopted to screen compounds with conditions of oral bioavailability $(\mathrm{OB}) \geq 30 \%$ [22] and druglike (DL) activity $\geq 0.18[23]$ and collect compounds-related targets.

In order to obtain more targets, simplified molecular input line entry specification (SMILES) information of bioactive compounds was obtained from the PubChem (https://pubchem.ncbi.nlm.nih.gov/) database and we imported SMILES information into the SwissTargetPrediction platform (http://www.swisstargetprediction.ch/), and the attribution was set as "homo sapiens" and probability $\geq 0.4$ to collect the target of the compound.

2.2. Collection of Targets of Asthma. The word "asthma” was used as a keyword in the GeneCards database (https://www. genecards.org/), Herb database (http://herb.ac.cn/), and DisGeNET database (https://www.disgenet.org) and the attribute was set to "homo sapiens" to obtain targets in different databases. Subsequently, all targets were uploaded to the online Venn diagram (https://bioinfogp.cnb.csic.es/ tools/venny/index.html) to obtain the common targets.

\subsection{Establishment of an SXC-Compounds-Target Protein} Network. The Uniprot database (https://www.uniprot.org/) was applied to normalize the names of target genes and convert it into abbreviations, and targets without corresponding gene names were deleted. Finally, the obtained chemical compounds and their corresponding targets were imported into Cytoscape (version 3.8.0 for Windows) software to construct a network visual diagram of SXC-compounds-targets.

2.4. Establishment of Protein Interaction Network. The targets of compounds and disease were uploaded to the online Venn diagram, respectively, to obtain their intersection genes, which data were sequentially uploaded to the STRING 11.0 database (https://string-db.org/). Species was set to "homo sapiens" and confidence level was set to $\geq 0.700$, respectively. After deleting the free target, a target-protein interaction network was constructed. Finally, the data were imported into Cytoscape software for visual analysis and network topology analysis, and key target analysis was performed through plugin CytoNCA to obtain the PPI network.

2.5. Analysis of $G O$ and KEGG Enrichment. GO biological function enrichment analysis and KEGG signaling pathway enrichment analysis on the key genes of PPI by using R (version 4.0.3 for Windows) and RStudio (version 1.4.1103 for Windows) software which install the packages of org.Hs.eg.db, ggplot2, enrichplot, and clusterProfiler [24]. To understand the complex relationships among compounds, targets, and pathways, compound-target-pathway networks were constructed and analyzed by Cytoscape.

2.6. Molecular Docking. In preparation for molecular docking, the three-dimensional (3D) protein structures of the key proteins in the PPI network and four active chemical ingredients were downloaded from the Protein Data Bank (PDB) database (http://www.rcsb.org/) and PubChem database, respectively. Then, budesonide was considered as a reference [25], and the SDF format of the compound was converted into the MOL2 format using Open Babel software (http://openbabel.org/wiki/Main_Page). After dehydration, hydrogenation, and compound pretreatment of the 
TABLE 1: The information of the bioactive compounds of SXC.

\begin{tabular}{|c|c|c|c|c|c|c|c|c|c|}
\hline No. & MOL ID & Compound & $\mathrm{OB}(\%)$ & $\mathrm{DL}$ & CAS & Molecular structure & $\begin{array}{l}\text { Molecular } \\
\text { formula }\end{array}$ & $\begin{array}{l}\text { Molecular weight (g/ } \\
\text { mol) }\end{array}$ & Reference \\
\hline 1 & MOL000006 & Luteolin & 36.16 & 0.25 & 491-70-3 & & $\mathrm{C}_{15} \mathrm{H}_{10} \mathrm{O}_{6}$ & 286.24 & {$[26]$} \\
\hline 2 & MOL001689 & Acacetin & 34.97 & 0.24 & $480-44-4$ & & $\mathrm{C}_{16} \mathrm{H}_{12} \mathrm{O}_{5}$ & 286.26 & {$[26]$} \\
\hline 3 & MOL000098 & Quercetin & 46.43 & 0.28 & $117-39-5$ & & $\mathrm{C}_{15} \mathrm{H}_{10} \mathrm{O}_{7}$ & 302.23 & {$[26]$} \\
\hline 4 & MOL002915 & Salvigenin & 49.07 & 0.33 & $\begin{array}{l}19103-54- \\
9\end{array}$ & & $\mathrm{C}_{18} \mathrm{H}_{16} \mathrm{O}_{6}$ & 328.30 & [19] \\
\hline 5 & MOL005573 & Genkwanin & 37.13 & 0.24 & $437-64-9$ & & $\mathrm{C}_{16} \mathrm{H}_{12} \mathrm{O}_{5}$ & 284.26 & [27] \\
\hline 6 & MOL004425 & Icariin & 41.58 & 0.61 & $489-32-7$ & & $\mathrm{C}_{33} \mathrm{H}_{40} \mathrm{O}_{15}$ & 676.70 & {$[16]$} \\
\hline 7 & MOL001987 & $\beta$-Sitosterol & 36.91 & 0.75 & $83-46-5$ & & $\mathrm{C}_{29} \mathrm{H}_{50} \mathrm{O}$ & 414.70 & {$[28]$} \\
\hline 8 & MOL001790 & Linarin & 39.84 & 0.71 & $480-36-4$ & & $\mathrm{C}_{28} \mathrm{H}_{32} \mathrm{O}_{14}$ & 592.50 & [29] \\
\hline
\end{tabular}

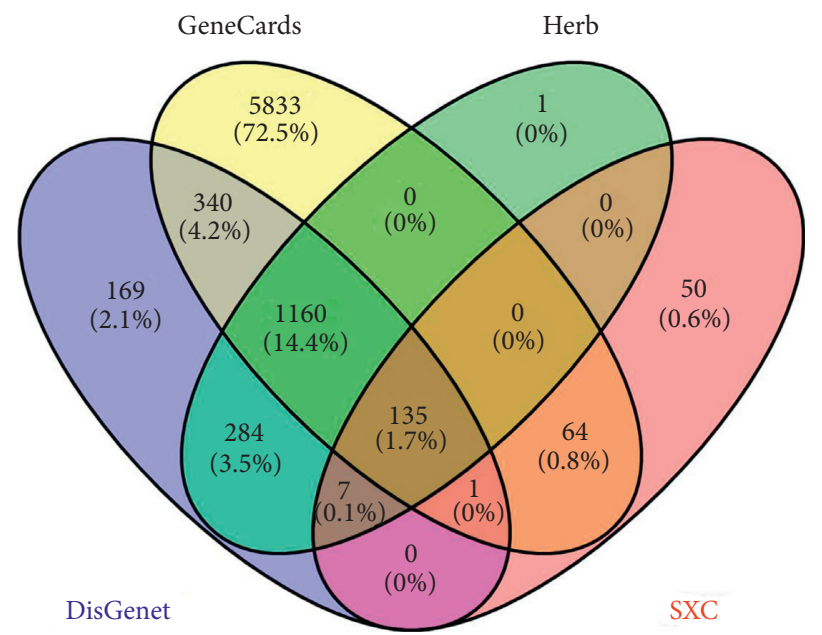

Figure 1: Venn diagram of targets of SXC and asthma.

downloaded proteins with AutoDock tools, AutoDock Vina (version 1.1.2 for Windows) software (http://vina.scripps. edu/) was used for molecular docking of the receptor and ligand. Finally, visualizing and analyzing the results of molecular docking conformation were completed by PyMOL (version 2.4.1 for Windows) software (https:// pymol.org/2/) and ProteinsPlus (https://proteins.plus/).

\section{Results and Discussion}

3.1. Active Compounds and Targets of SXC. By a literature search, the composition database of SXC was established; all compounds were screened by the TCMSP database for
$\mathrm{OB} \geq 30 \%$ and $\mathrm{DL} \geq 0.18$. To further discover the targets, the corresponding targets of these bioactive compounds information were supplemented with SwissTargetPrediction database. After deleting the duplicate targets, a total of 257 potential targets and 8 bioactive compounds were found (Table 1).

3.2. Asthma-Related Targets and Common Targets. The DisGeNET database screened 2096 targets, the GeneCards database screened 7533 targets, and the Herb database screened 1587 targets. Gene names of the above targets were included in the online Venn diagram and a total of 1295 


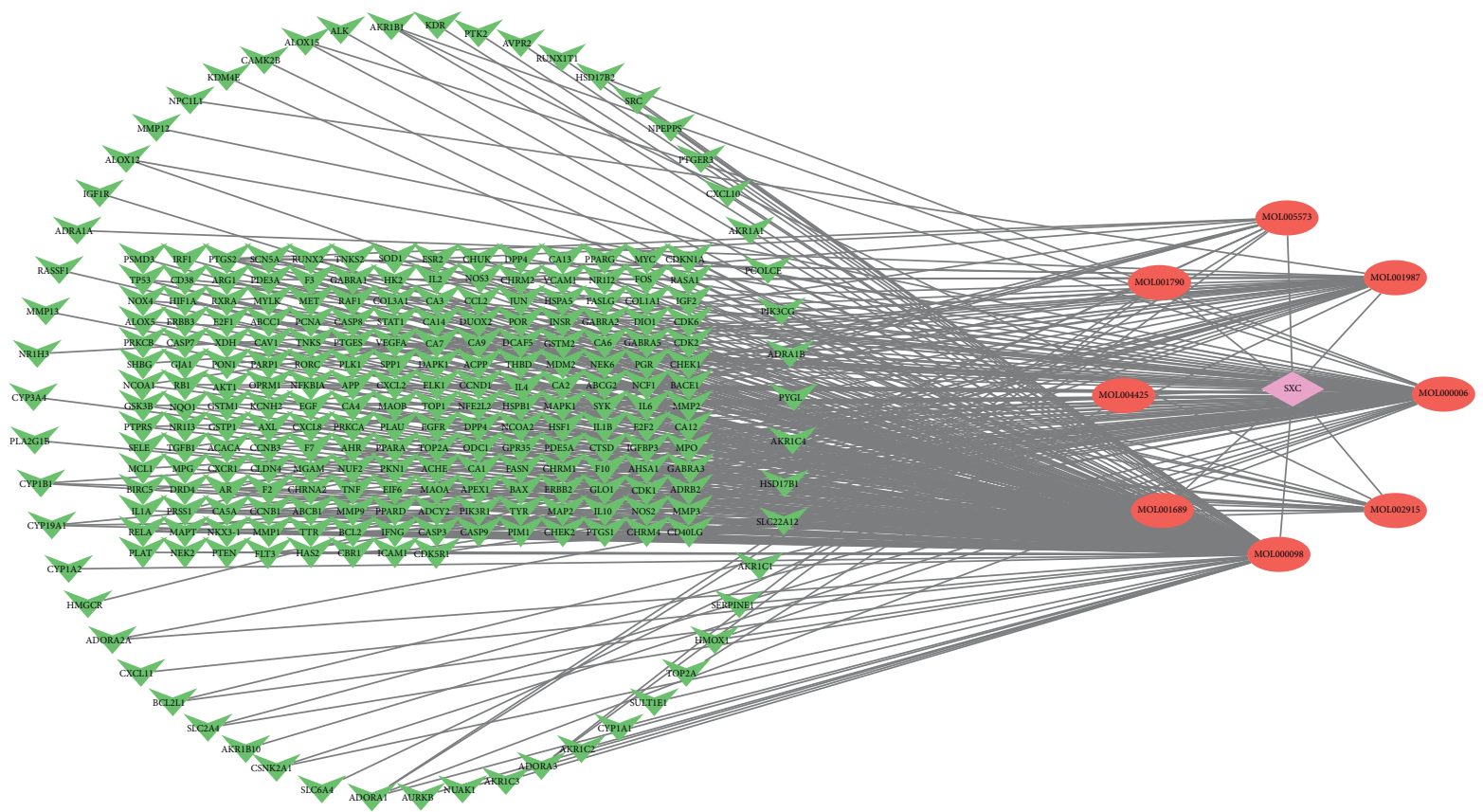

FIGURE 2: SXC-compounds-target network (red ellipses represent compounds, and green V represents compounds, and pink diamond represents SXC).

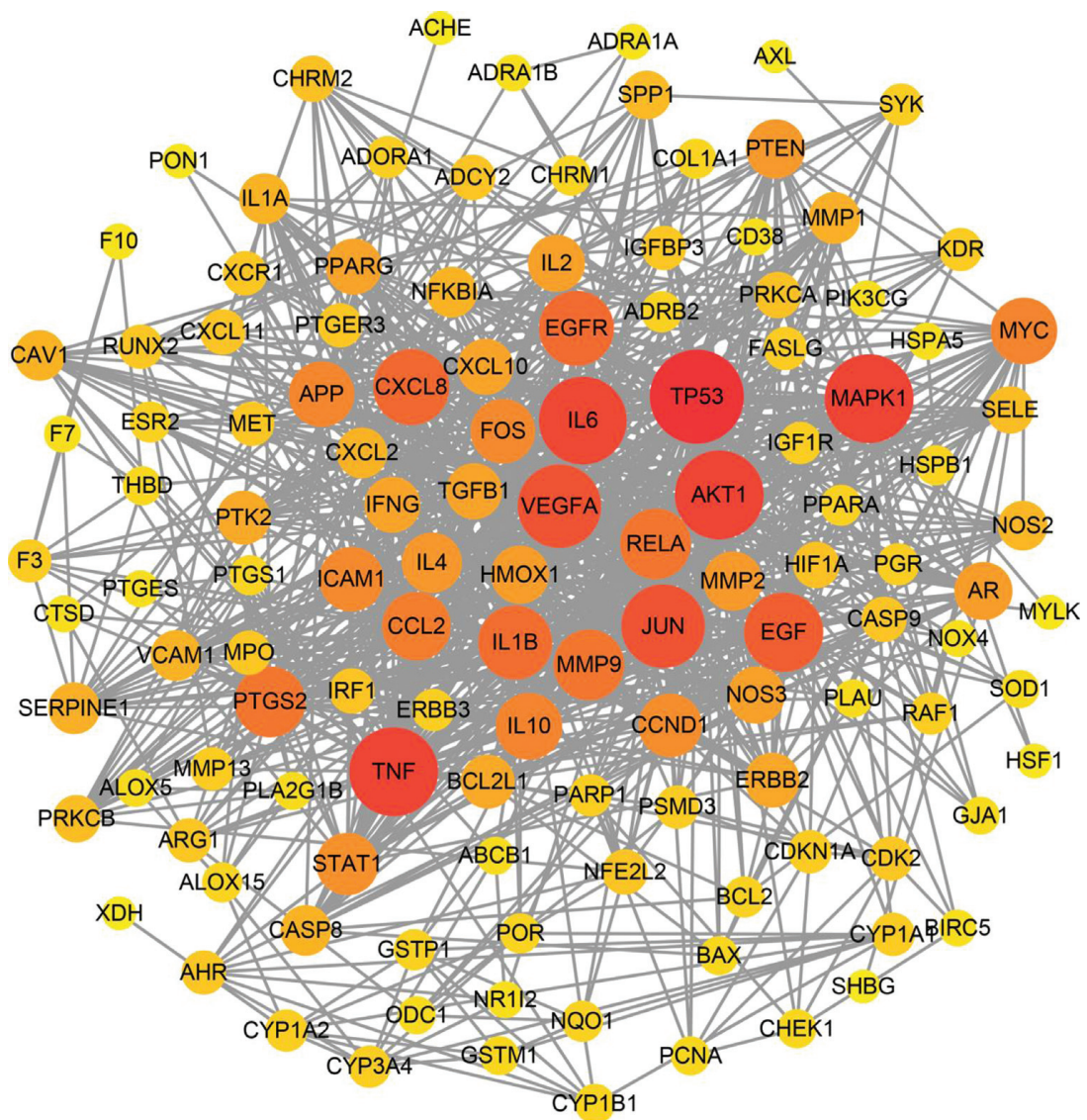

FIGURE 3: The protein-protein interaction (PPI) network (the size and color saturation of the node were proportional to its significance). 


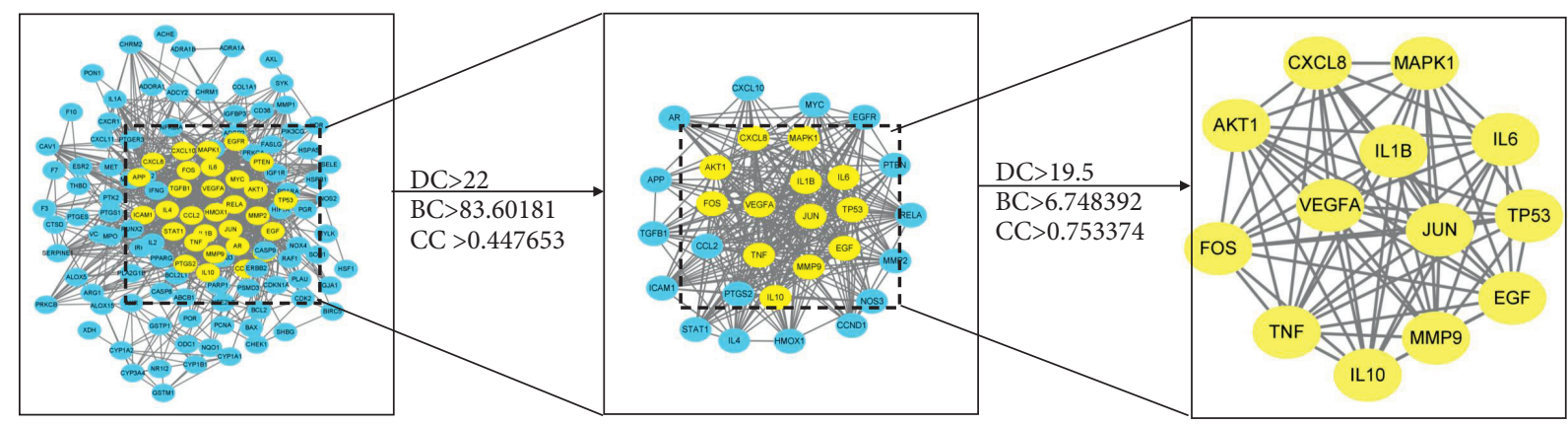

FIGURE 4: Key targets screening process.

TABLe 2: Key targets of PPI network.

\begin{tabular}{lcccc}
\hline No. & Target & Degree & Betweenness & Closeness \\
\hline 1 & IL-6 & 12.00 & 1.52 & 1.00 \\
2 & EGF & 12.00 & 1.52 & 1.00 \\
3 & JUN & 12.00 & 1.52 & 1.00 \\
4 & TNF & 12.00 & 1.52 & 1.00 \\
5 & IL10 & 11.00 & 0.82 & 0.92 \\
6 & MMP9 & 11.00 & 1.09 & 0.92 \\
7 & CXCL8 & 11.00 & 0.82 & 0.92 \\
8 & MAPK1 & 11.00 & 1.09 & 0.92 \\
9 & AKT1 & 11.00 & 1.07 & 0.92 \\
10 & VEGFA & 10.00 & 0.89 & 0.86 \\
11 & FOS & 10.00 & 0.89 & 0.86 \\
12 & IL1B & 10.00 & 0.62 & 0.86 \\
13 & TP53 & 9.00 & 0.62 & 0.80 \\
\hline
\end{tabular}

asthma-related intersection targets was obtained. Then, asthma-related targets and targets of active compounds were uploaded to the online Venn diagram and 135 common genes were obtained between SXC and asthma (Figure 1).

\subsection{Construction and Analysis of SXC-Compounds-Target} Network. A total of 8 active chemical compounds of SXC and their corresponding targets were imported into Cytoscape software, and the SXC-compound-target network relationship diagram (Figure 2) was obtained. Figure 2 shows that the diagram included a total of 268 nodes and 407 edges. The results indicated that quercetin $($ degree $=214.0), \quad$ luteolin $\quad($ degree $=92.0), \quad \beta$-sitosterol $($ degree $=37.0), \quad$ acacetin $\quad($ degree $=29.0), \quad$ salvigenin $($ degree $=15.0)$, and genkwanin $($ degree $=13.0)$ may be had relationship in the treatment of asthma.

3.4. PPI Network Construction and Topology Analysis. After importing the common targets to the STRING database, the subnetworks that cannot be connected to the free target and the main network were deleted. The PPI network was imported into Cytoscape software for key target analysis, and 125 nodes and 983 edges were obtained (Figure 3 ). Then, CytoNCA plug-in was performed to obtain all targets. The top 5 targets in degree value were IL-6 (degree =12.0), EGF $($ degree $=12.0)$, JUN $($ degree $=12.0)$, TNF $($ degree $=12.0)$, and IL-10 (degree $=11.0)$. The average degree of node is 3.03 and average of closeness is 0.40 , and the average of betweenness is 405.97. Colors and size indicated that the importance of target in the network. If the degree (DC) and betweenness (BC) were greater than twice the median value and if closeness (CC) was greater than the median value to screen key targets [30], construction and topology analysis of the PPI network was performed as shown in Figure 4 and Table 2.

\subsection{Analysis of GO Function Enrichment and KEGG Pathway} Enrichment. The key targets were analyzed by RStudio software, and a total of $1677 \mathrm{GO}$ entries with $P<0.05$ were enriched (Figure 5). This involved 1606 biological process (BP) that included cellular responses to reactive oxygen species (ROS) and cellular responses to oxidative stress and chemical stress. Furthermore, 9 cellular components (CC) were found to contain a platelet alpha granule lumen and a secretory granule lumen. In addition, 62 molecular functions (MF) were found that mainly include receptor ligand activity, signal receptor activator activity, and cytokine receptor binding.

A total of 110 pathways with $P<0.05$ were enriched by KEGG pathway enrichment, and Figure 5 showed the bubble chart of the top 20 KEGG signaling pathway enrichment analysis. The asthma-related pathways mainly included advanced glycation end products (AGE)-receptor for advanced glycation end products (RAGE) signaling pathway, IL-17 signaling pathway, TNF signaling pathway, and MAPK signaling pathway. In addition, other diseases were involved, such as Chagas disease, whooping cough, Yersinia infection, and hepatitis B.

3.6. Compounds-Target-Pathway Network. As shown in Figure 6, Cytoscape was used to construct a compoundstargets-pathway network. The network consisted of 38 nodes and 183 edges, including 4 active compounds, 13 targets, and 20 KEGG pathways. These findings indicate that SXC acts on multiple pathways through multiple targets of multiple compounds to achieve a therapeutic effect on asthma.

\subsection{Diagram of the Treatment Pathway of SXC. KEGG} Mapper was applied to display the key targets in the pathways that were most related to asthma. As shown in Figure 7, red squares represent pathway network multiple nodes that are targets of the compounds that can be 
cellular response to reactive oxygen species cellular response to oxidative stress cellular response to chemical stress response to reactive oxygen species cellular response to biotic stimulus regulation of DNA-binding transcription factor activity response to oxidative stress response to lipopolysaccharide response to molecule of bacterial origin cellular response to lipopolysaccharide RNA polymerase II transcription regulator complex platelet alpha granule lumen secretory granule lumen cytoplasmic vesicle lumen vesicle lumen platelet alpha granule transcription regulator complex ficolin-1-rich granule ficolin-1-rich granule lumen pseudopodium cytokine activity receptor ligand activity signaling receptor activator activity cytokine receptor binding growth factor receptor binding growth factor activity R-SMAD binding protein phosphatase $2 \mathrm{~A}$ binding phosphatase binding serine/threonine/tyrosine kinase activity
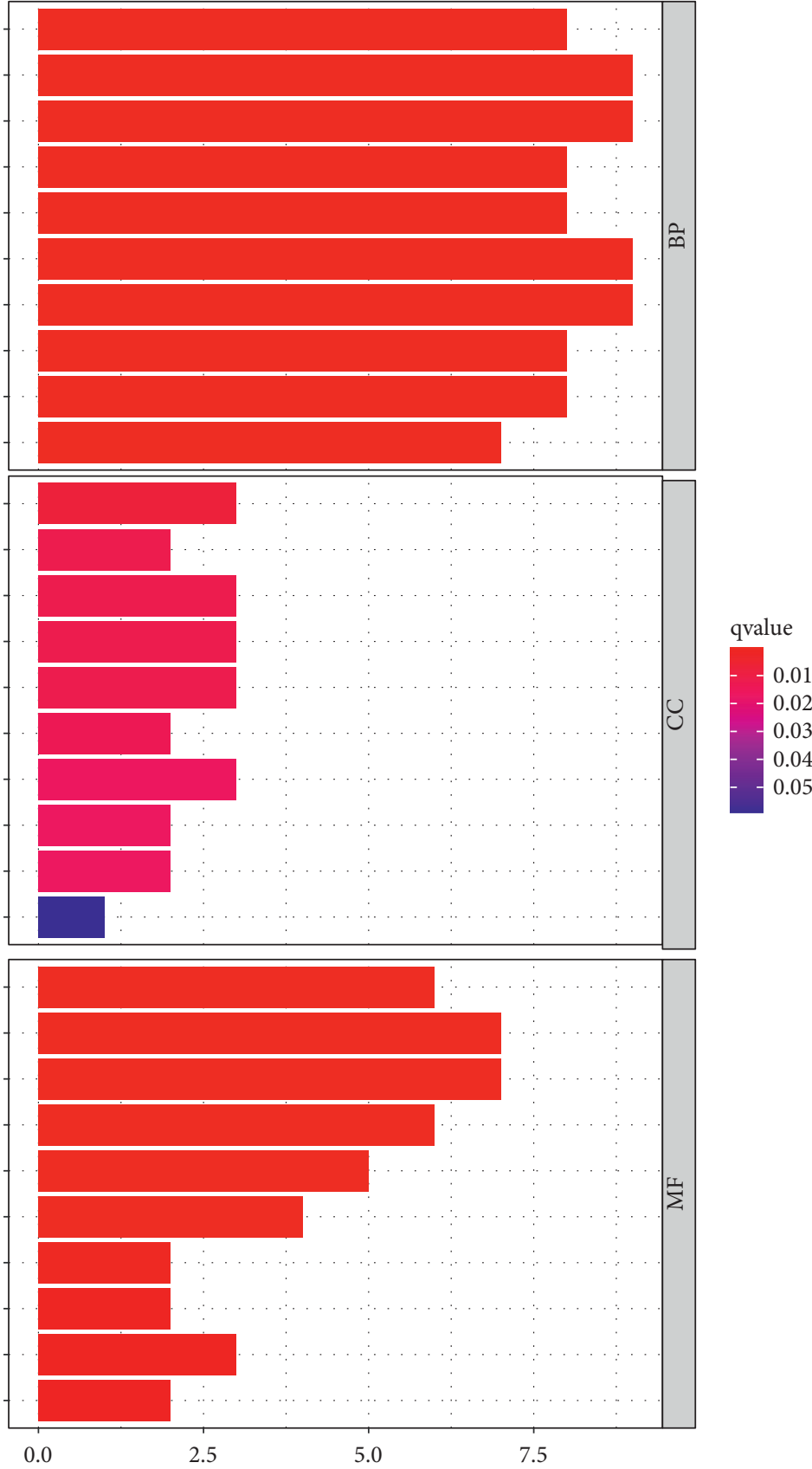

(a)

Figure 5: Continued. 


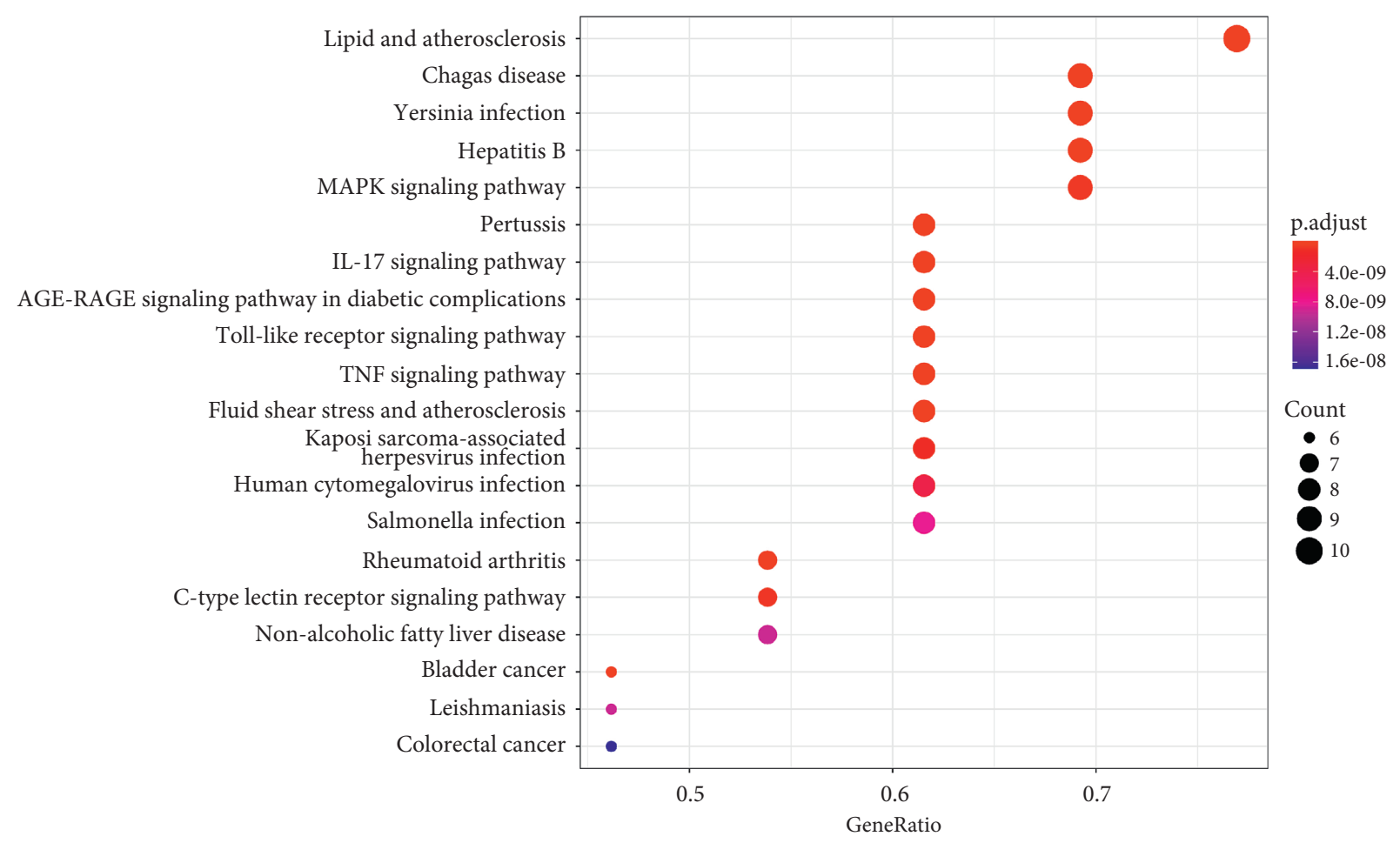

(b)

FIGURE 5: GO and KEGG pathway enrichment analyses.

regulated by quercetin, luteolin, and $\beta$-sitosterol. All abovementioned results demonstrated that the active ingredients of SXC achieved effects through multiple targets and pathways and synergistic effects.

3.8. Molecular Docking. The top 4 active ingredients with a degree value of SXC were selected as ligands, while 13 core targets of the PPI network were used as receptors. To further validate the SXC compounds against asthma, molecular docking was carried out using the abovementioned ligands and receptors. The results of molecular docking showed that luteolin, quercetin, acacetin, and $\beta$-sitosterol all bound well to TNF, MMP9, and AKT1 (Figure 8 and Table 3).

\section{Discussion}

SXC from Xinjiang, China, has a long-term effect and shown significant treatment efficacy and regional and national features to treating certain diseases. For example, Loki Zupa Decoction [31] and Hanchuan Zupa Granule [32] both are related to SXC for the treatment of asthma. Network pharmacology results showed that 4 active ingredients of SXC, most of them belonging to flavonoids, may be related to the underlying antiasthmatic mechanism of action. Furthermore, in previous studies, it was shown that flavonoids have antitumor, antioxidation, anti-inflammatory, and anticoagulation effects and improve sugar and lipid metabolism [33]. The results of the compounds-targetpathway diagram demonstrated that quercetin, luteolin, $\beta$-sitosterol, and acacetin highly correlated with antiasthma effects.

As a natural flavonoid with polyphenolic hydroxyl groups, luteolin can alleviate the inflammatory response in a variety of ways [34] and reduce the production of reactive oxygen and reactive nitrogen, thereby affecting the metabolism of arachidonic acid and various inflammatory signaling pathways [35] and inhibiting the expression of inflammatory cytokines and inflammatory mediators [36]. Quercetin is a flavonol compound that is widely distributed in plants, vegetables, and fruits [37] and has a variety of biological activities against cardiovascular diseases, cancer, and diabetes [38]. Several studies have shown that the effect of anti-inflammatory and antiasthma by quercetin has been verified [39-41]. In addition, in a previous report, it was demonstrated that quercetin can improve beta-agonist-induced relaxation and relax airway smooth muscle [42], which is similar to our previous study results. Moreover, in recent reports, it was shown that acacetin has anti-inflammatory [43, 44] and antiasthma [45] activity in lipopolysaccharide (LPS), D-galactosamine, and ovalbumin-induced damage. Its mechanism of action may involve inhibition of the expression of iNOS and COX-2 and phosphorylation of I $\kappa \mathrm{B} \alpha$ [46], weakening TNF- $\alpha$, IL-6, and TLR 4 levels and inhibiting the phosphorylation of kinases and expression of $\mathrm{NF}-\kappa \mathrm{B}$ [47].

As shown by the analysis results of the PPI network topology, 13 key targets were screened, and most were inflammatory cytokines, it is worth to note that IL-6, TNF- $\alpha$, and IL-10 play an important role in the development of 


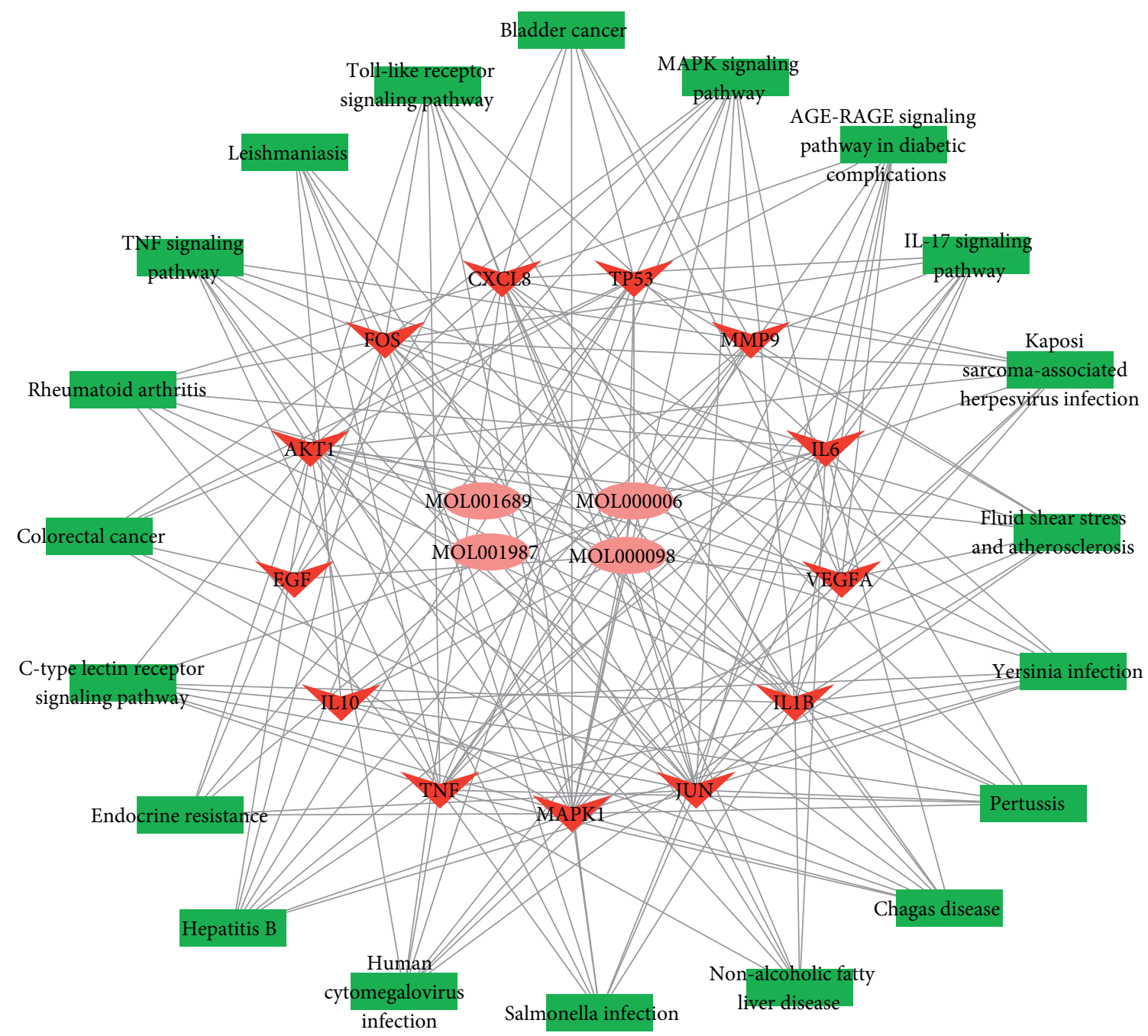

FIGURE 6: Network of active compounds-targets-pathway (pink ellipses represent compounds, red V-grooves represent target, and green rectangles represent pathways).

asthma. IL-6 is a cytokine that is activated and secreted by lymphocytes and mononuclear macrophages and has an important impact on the development of asthma [48]. Furthermore, IL-6 receptor signaling regulates the expression of Th17-related genes by activating STAT3 to induce the development of Th17 cells [49]. IL-10 is a multicell-derived, multifunctional anti-inflammatory cytokine that is involved in the regulation of cell growth and differentiation as well as in inflammatory and immune responses [50]. In addition, IL-10 plays a key role in controlling immune responses. As recognized inflammatory and immunosuppressive factors, the TNF family plays an important role in the pathogenesis of asthma and inflammation of other tissues [51].

The GO enrichment data showed that SXC mainly plays a role in the responses of cells to ROS, oxidative stress, chemical stress, and LPS. Obviously, there is an obvious synergy between the different signaling pathways with the enrichment of the KEGG pathway, such as TLR signaling pathway, TNF signaling pathway, and MAPK signaling pathway. It is worth noting that the MAPK signaling pathway is an important pathway involved in the pathogenesis and progression of bronchial asthma [52]. Related regulatory factors of the MAPK signaling pathway include c-Jun N-terminal kinase (JNK), p38, and extracellular signal-regulated kinase (ERK) [53]. Furthermore, the MAPK signaling pathway can control the expression of inflammatory factors (IL-6 and TNF- $\alpha$ ) to regulate inflammation and immune responses in asthma $[54,55]$. It is reported that the ethyl acetate extract of SXC can significantly reduce the levels of ET-1, IL-2, IL-6, and TNF- $\alpha$ in the serum of asthmatic rats in a dose-dependent trend [56]. Hou et al. [57] observed the effect of SXC on cytokines in allergic asthma mice and found that after SXC administration, the levels of IL-4 and IL-17 in BALF of asthma mice were significantly reduced, and IFN- $\gamma$ level was significantly increased. 


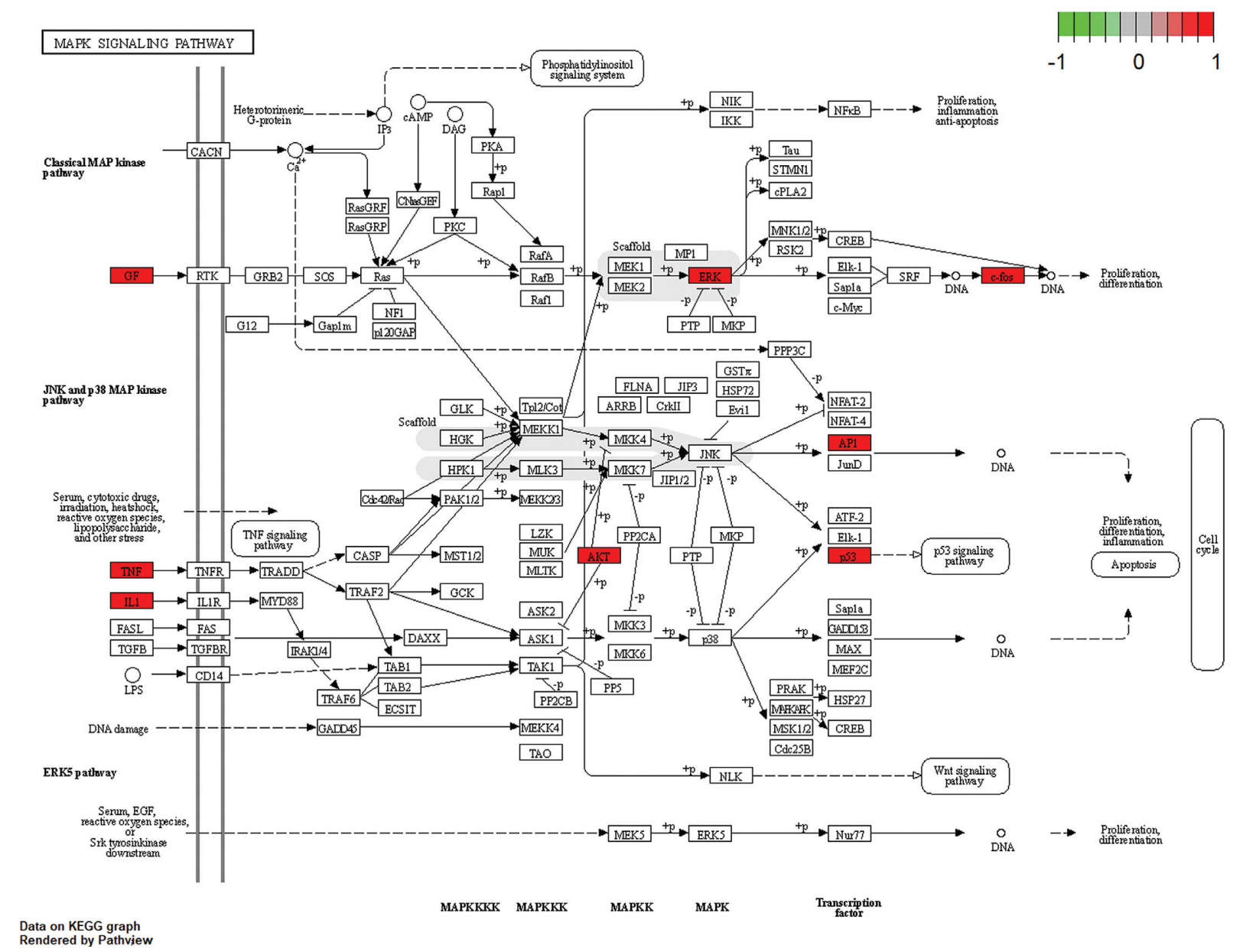

FIGURE 7: MAPK signaling pathway enrichment analysis (red rectangles represent important targets).

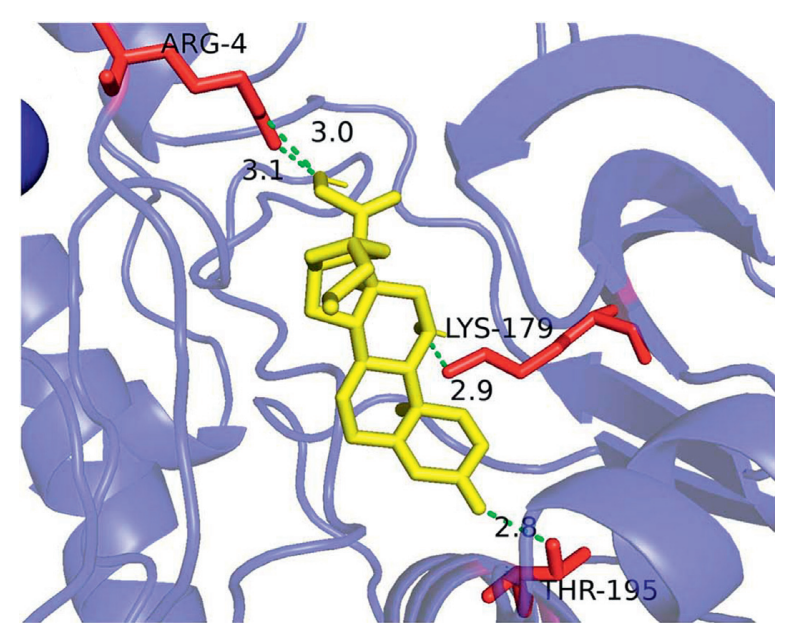

(a)

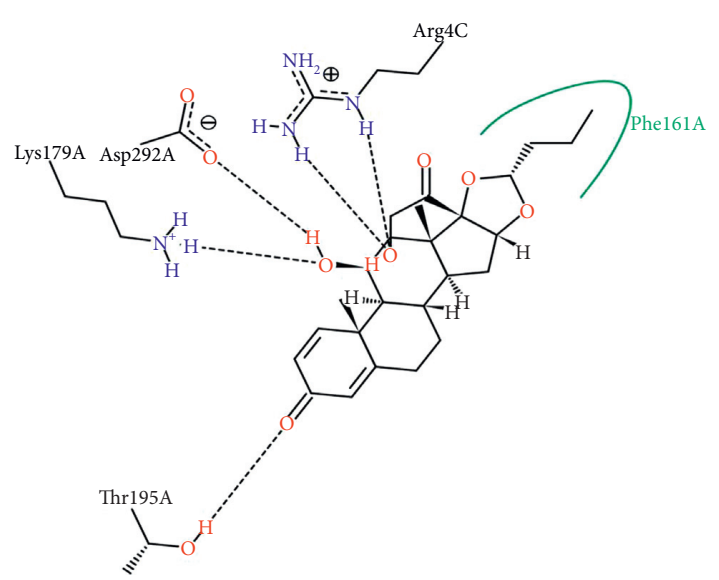

(b)

Figure 8: Continued. 

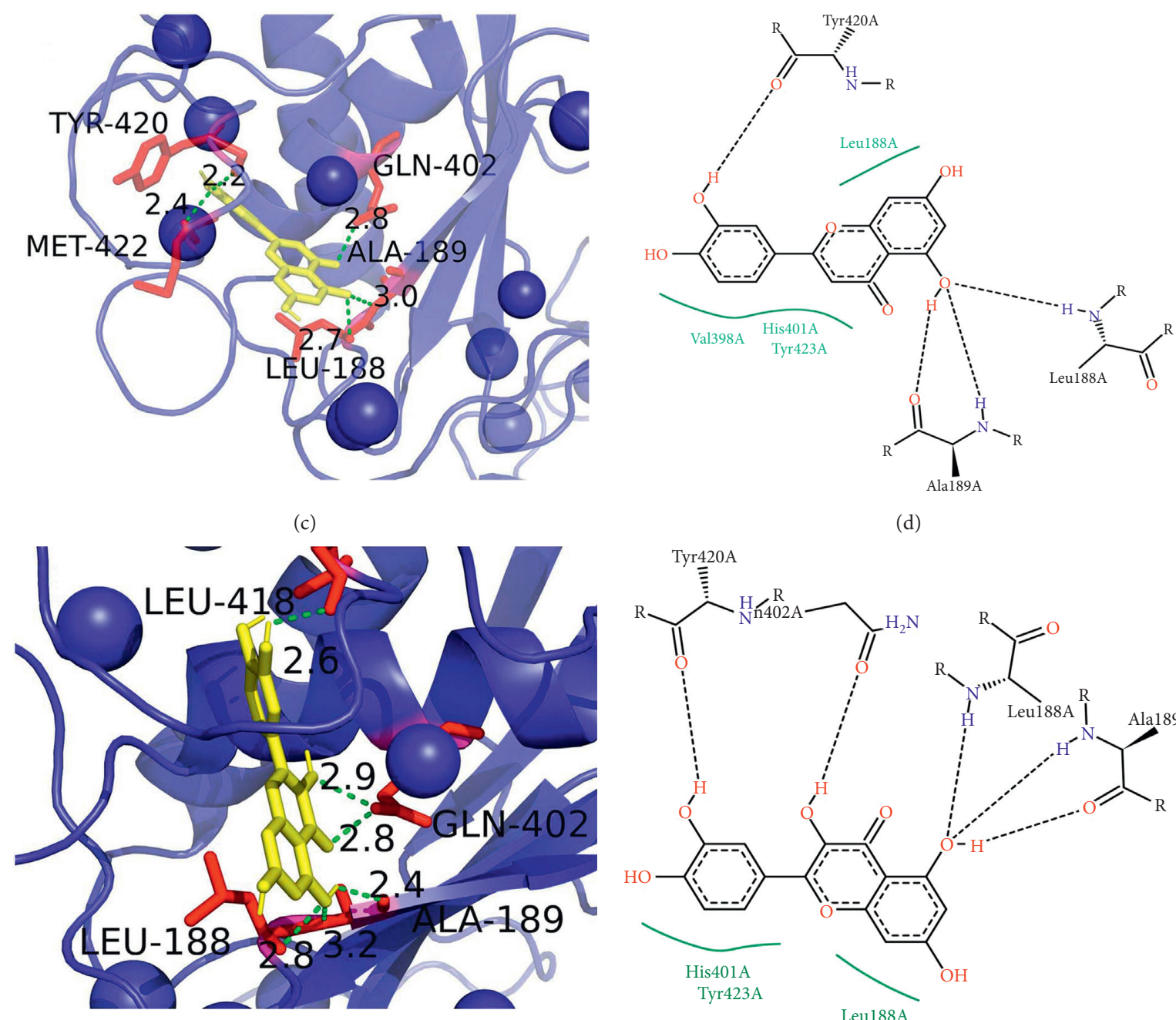

(d)

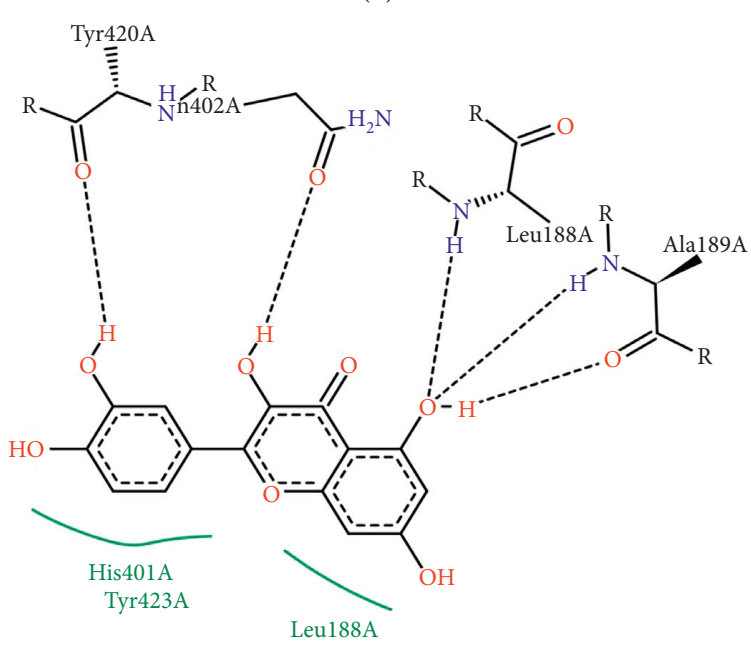

(e)

(f)
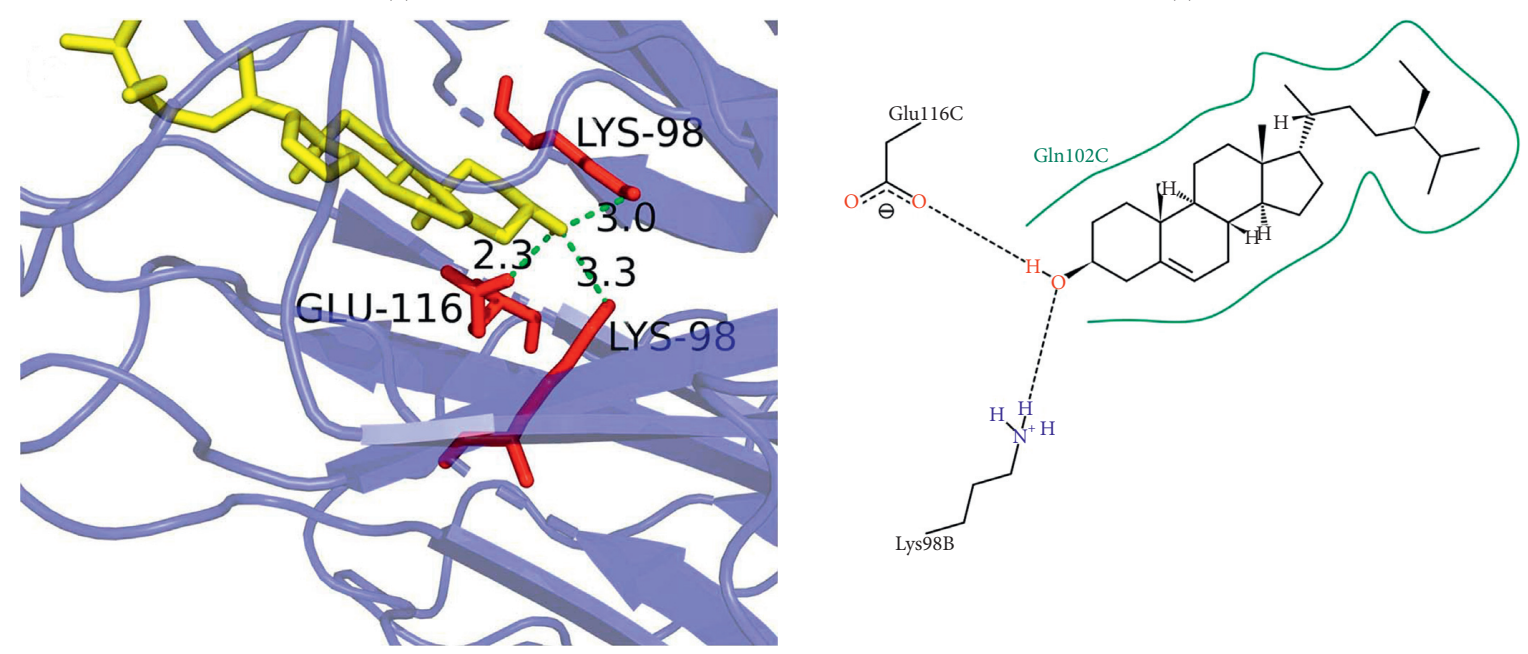

(g)

Figure 8: Continued. 


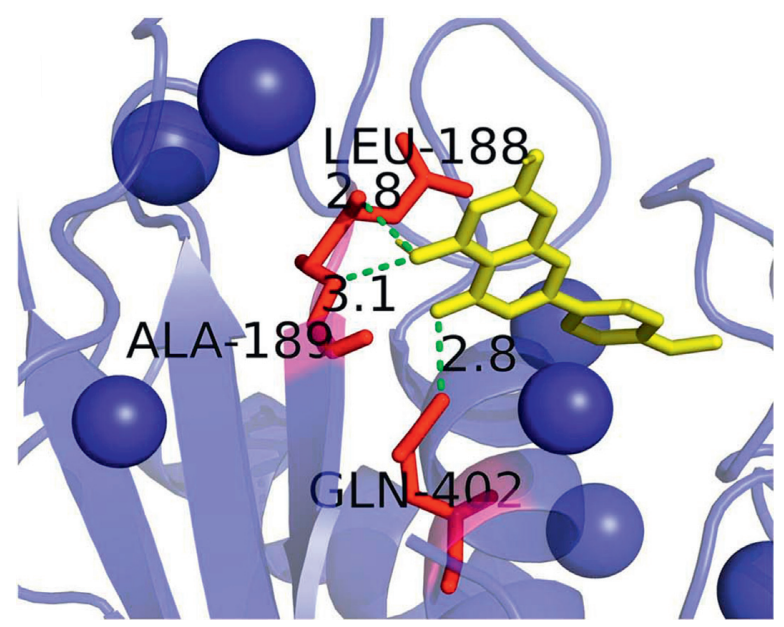

(i)

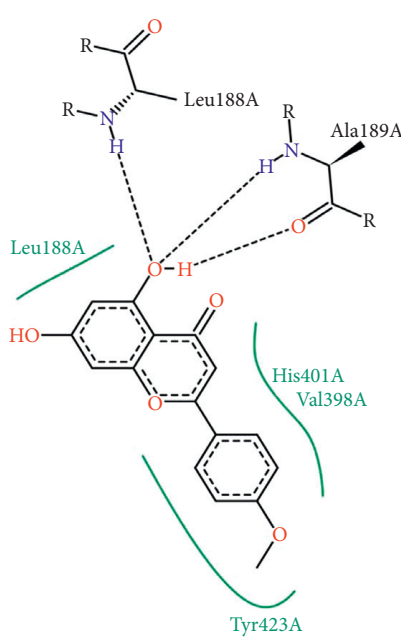

(j)

Figure 8: Docking binding energy between ligand and protein. (a, b) Budesonide-AKT1. (c, d) Luteolin-MMP9. (e, f) Quercetin-MMP9. (g, h) Beta-sitosterol-TNF. (I, j) Acacetin-MMP9.

TABle 3: Binding affinity of the compounds and key targets by molecular docking.

\begin{tabular}{|c|c|c|c|c|c|c|c|}
\hline No. & Compound & Molecular structure & Target & PDB ID & Affinity $(\mathrm{kcal} / \mathrm{mol})$ & Polar contact & Center $(X, Y, Z)$ \\
\hline \multirow[t]{2}{*}{1} & \multirow{2}{*}{ Budesonide } & & \multirow{2}{*}{ AKT1 } & \multirow{2}{*}{ 3CQW } & \multirow{2}{*}{-9.5} & $\begin{array}{l}\text { ARG-4 } \\
\text { LYS-179 }\end{array}$ & $\begin{array}{c}2.68 \\
-0.87\end{array}$ \\
\hline & & & & & & Thr-195 & 26.01 \\
\hline 2 & Luteolin & & MMP9 & 2OW0 & -10.5 & $\begin{array}{l}\text { LEU-188 } \\
\text { ALA-189 } \\
\text { GLN-402 } \\
\text { TYR-420 } \\
\text { MET-422 }\end{array}$ & $\begin{array}{l}46.89 \\
12.14 \\
49.07\end{array}$ \\
\hline \multirow[t]{2}{*}{3} & \multirow[t]{2}{*}{ Quercetin } & & \multirow[t]{2}{*}{ MMP9 } & \multirow[t]{2}{*}{ 2OW0 } & \multirow[t]{2}{*}{-10.5} & $\begin{array}{l}\text { LEU-188 } \\
\text { ALA-189 }\end{array}$ & $\begin{array}{l}46.89 \\
12.14\end{array}$ \\
\hline & & & & & & GLN-402 & 49.07 \\
\hline \multirow[t]{2}{*}{4} & \multirow[t]{2}{*}{ Beta-sitosterol } & & \multirow[t]{2}{*}{ TNF } & \multirow[t]{2}{*}{$1 \mathrm{TNF}$} & \multirow[t]{2}{*}{-9.5} & $\begin{array}{c}\text { LEU-418 } \\
\text { LYS-98 }\end{array}$ & $\begin{array}{l}19.97 \\
49.68\end{array}$ \\
\hline & & & & & & GLU-116 & 39.93 \\
\hline \multirow[t]{2}{*}{5} & \multirow[t]{2}{*}{ Acacetin } & & \multirow[t]{2}{*}{ MMP9 } & \multirow[t]{2}{*}{ 2OW0 } & \multirow[t]{2}{*}{-10.2} & $\begin{array}{l}\text { ALA-189 } \\
\text { GLN-402 }\end{array}$ & $\begin{array}{l}46.89 \\
12.14\end{array}$ \\
\hline & & & & & & LEU-188 & 49.07 \\
\hline
\end{tabular}

In this study, molecular docking was carried out to investigate the binding ability between the compounds and targets. The smaller the value of binding energy, the better binding between the compound and target; the results of molecular docking showed that the binding energy of receptor and ligand were both $<-5.0 \mathrm{kcal} / \mathrm{mol}$.

\section{Conclusions}

In summary, the results of network pharmacology showed that Hyssopus cuspidatus Boriss. has multiple compounds, multiple targets, and multiple pathways to synergistically play a role in treating asthma. These findings point out the direction and provide scientific evidence for subsequent clinical and experimental research. However, considering the certain limitations in network pharmacology of the databases updates and different target prediction mechanisms, it is still necessary to verify the pathway and target through the experiments in the future to confirm the accuracy of the prediction results of this study.

\section{Data Availability}

The data used to support the findings of this study are available from the corresponding author upon request. 


\section{Ethical Approval}

No animals/humans were used for the studies that are the basis of this research.

\section{Disclosure}

Rongchang Liu and Yan Mao are the co-first authors.

\section{Conflicts of Interest}

The authors declare that there are no conflicts of interest regarding the publication of this paper.

\section{Acknowledgments}

This work was supported by National Natural Science Foundation of China (grant no. 81760709) and Basic Research Business Fund Project of Public Welfare Research Institutes in Xinjiang Uygur Autonomous Region, China (grant no. KY2020081).

\section{References}

[1] R. C. Murphy and T. S. Hallstrand, "Exploring the origin and regulatory role of mast cells in asthma," Current Opinion in Allergy \& Clinical Immunology, vol. 21, no. 1, pp. 71-78, 2021.

[2] I. H. Heijink, V. N. S. Kuchibhotla, M. P. Roffel et al., "Epithelial cell dysfunction, a major driver of asthma development," Allergy, vol. 75, no. 8, pp. 1902-1917, 2020.

[3] F. Xiu, W. Cheng, Y. Jiang et al., "A network pharmacologybased strategy for predicting anti-inflammatory targets of ephedra in treating asthma," International Immunopharmacology, vol. 83, Article ID 106423, 2020.

[4] A. A. Ramadan, J. M. Gaffin, E. Israel, and W. Phipatanakul, "Asthma and corticosteroid responses in childhood and adult asthma," Clinics in Chest Medicine, vol. 40, no. 1, pp. 163-177, 2019.

[5] I. Henderson, E. Caiazzo, C. McSharry, T. J. Guzik, and P. Maffia, "Why do some asthma patients respond poorly to glucocorticoid therapy?" Pharmacological Research, vol. 160, Article ID 105189, 2020.

[6] S. E. Reese, C. J. Xu, H. T. den Dekker et al., "Epigenome-wide meta-analysis of DNA methylation and childhood asthma," The Journal of Allergy and Clinical Immunology, vol. 143, no. 6, pp. 2062-2074, 2019.

[7] I. V. Yang, C. A. Lozupone, and D. A. Schwartz, "The environment, epigenome, and asthma," The Journal of Allergy and Clinical Immunology, vol. 140, no. 1, pp. 14-23, 2017.

[8] K. W. Huang, T. Yang, Y. Xu et al., "Prevalence, risk factors, and management of asthma in China: a national cross-sectional study," The Lancet, vol. 394, no. 10196, pp. 407-418, 2019.

[9] X. Li, P. Song, Y. Zhu et al., "The disease burden of childhood asthma in China: a systematic review and meta-analysis," Journal of Global Health, vol. 10, no. 1, Article ID 010801, 2020.

[10] P. Liang, S. Peng, M. Zhang, Y. Ma, X. Zhen, and H. Li, "Huai Qi Huang corrects the balance of Th1/Th2 and Treg/Th17 in an ovalbumin-induced asthma mouse model," Bioscience Reports, vol. 37, no. 6, 2017.

[11] J. Hong, S. Kim, and Y. Lee, "The ethanol extract of larvae, containing fatty acids and amino acids, exerts anti-asthmatic effects through inhibition of the GATA-3/Th2 signaling pathway in asthmatic mice," Molecules, vol. 24, no. 5, 2019.

[12] S.-Y. Lee, B. Kang, S.-H. Bok, S. S. Cho, and D.-H. Park, "Macmoondongtang modulates Th1-/Th2-related cytokines and alleviates asthma in a murine model," PLoS One, vol. 14, no. 12, Article ID e0224517, 2019.

[13] Y.-Y. Qiu, Y. Wu, M.-J. Lin, T. Bian, Y.-L. Xiao, and C. Qin, "LncRNA-MEG3 functions as a competing endogenous RNA to regulate Treg/Th17 balance in patients with asthma by targeting microRNA-17/ROR $\gamma \mathrm{t}$," Biomedicine \& Pharmacotherapy, vol. 111, pp. 386-394, 2019.

[14] R. Malaviya, J. D. Laskin, and D. L. Laskin, "Anti-TNF $\alpha$ therapy in inflammatory lung diseases," Pharmacology \& Therapeutics, vol. 180, pp. 90-98, 2017.

[15] T. Schmit, S. Ghosh, R. K. Mathur et al., "IL-6 deficiency exacerbates allergic asthma and abrogates the protective effect of allergic inflammation against Streptococcus pneumoniae pathogenesis," The Journal of Immunology, vol. 205, no. 2, pp. $469-479,2020$.

[16] O. Shomirzoeva, J. Li, S. Numonov, S. Atolikshoeva, and H. A. Aisa, "Chemical components of Hyssopus cuspidatus Boriss.: isolation and identification, characterization by HPLC-DAD-ESI-HRMS/MS, antioxidant activity and antimicrobial activity," Natural Product Research, vol. 34, no. 4, pp. 534-540, 2020.

[17] L. Zhao, Z. Ji, K. Li, B. Wang, Y. Zeng, and S. Tian, "HPLCDAD analysis of Hyssopus Cuspidatus Boriss extract and mensuration of its antioxygenation property," BMC Complementary Medicine and Therapies, vol. 20, no. 1, p. 228, 2020.

[18] F. Yuan, Y. Sun, H. Hasimu, J. He, and Y. Mao, "Effects of the different elutions of Hyssopus cuspidatus on smooth muscle contraction of isolated tracheal in Guinea pigs," China Pharmacy, vol. 39, no. 4, pp. 809-812, 2017.

[19] F. Yuan, R. Liu, M. Hu et al., "JAX2, an ethanol extract of Hyssopus cuspidatus Boriss, can prevent bronchial asthma by inhibiting MAPK/NF- $\kappa \mathrm{B}$ inflammatory signaling," Phytomedicine, vol. 57, pp. 305-314, 2019.

[20] F. Yuan, M. Hu, H. Hasimu et al., "Extract of Hyssopus cuspidatus in preventing and treating bronchial asthma of mechanism," Chinese Traditional Patent Medicine, vol. 39, no. 4, pp. 809-812, 2017.

[21] H. Li, N. Zhao, K. Yang, Z. Liu, and Q. Wang, "Chemical composition and toxicities of the essential oil derived from Hyssopus cuspidatus flowering aerial parts against Sitophilus zeamais and Heterodera avenae," Journal of Medicinal Plants Research, vol. 7, no. 7, pp. 343-348, 2013.

[22] H. Duan, G. J. Khan, L.-J. Shang et al., "Computational pharmacology and bioinformatics to explore the potential mechanism of Schisandra against atherosclerosis," Food and Chemical Toxicology, vol. 150, Article ID 112058, 2021.

[23] H. Duan, K.-F. Zhai, G. J. Khan et al., "Revealing the synergistic mechanism of multiple components in compound fengshiding capsule for rheumatoid arthritis therapeutics by network pharmacology," Current Molecular Medicine, vol. 19, no. 4, pp. 303-314, 2019.

[24] G. Yu, L.-G. Wang, Y. Han, and Q.-Y. He, "ClusterProfiler: an $\mathrm{R}$ package for comparing biological themes among gene clusters," OMICS: A Journal of Integrative Biology, vol. 16, no. 5, pp. 284-287, 2012.

[25] S. P. Peters, E. R. Bleecker, G. W. Canonica et al., "Serious asthma events with budesonide plus formoterol vs. Budesonide alone," New England Journal of Medicine, vol. 375, no. 9, pp. 850-860, 2016. 
[26] M. Wei and X. Sun, "Simultaneous determination of four constituents in Hyssopus cuspidatus by RP-HPLC," Chinese Journal of Experimental Traditional Medical Formulae, vol. 22, no. 11, pp. 77-79, 2016.

[27] M. Furukawa, M. Makino, E. Ohkoshi, T. Uchiyama, and Y. Fujimoto, "Terpenoids and phenethyl glucosides from Hyssopus cuspidatus (Labiatae)," Phytochemistry, vol. 72, no. 17, pp. 2244-2252, 2011.

[28] J. Zhao, F. Xu, J. He, W. Yan, Z.-Y. Gu, and L. Ma, "Study on chemical constituents of Hyssopus cuspidatus," Zhong Yao Cai, vol. 36, no. 1, pp. 54-57, 2013.

[29] X. Cai, A. Maimaiti, X. Wang, and J. He, "Chemical constituents of anti-asthmatic active site from overground part of Hyssopus cuspidatus," Journal of Chinese Medicinal Materials, vol. 44, no. 4, pp. 848-852, 2021.

[30] Z. Qu, S. Wu, Y. Zheng et al., "Predictive analysis of quality markers of Bupleurum scorzonerifolium based on fingerprint and network pharmacology," Chinese Traditional and Herbal Drugs, vol. 52, no. 9, pp. 2678-2686, 2021.

[31] Y. Wei, M. Abduwaki, M. Li et al., "Loki zupa (Luooukezupa) decoction reduced airway inflammation in an OVA-induced asthma mouse model," Chinese Medicine, vol. 11, no. 1, p. 22, 2016.

[32] P. Li, J. Wang, C. Wang et al., "Therapeutic effects and mechanisms study of Hanchuan Zupa Granule in a Guinea pig model of cough variant asthma," Journal of Ethnopharmacology, vol. 269, Article ID 113719, 2021.

[33] T. Tanaka and R. Takahashi, "Flavonoids and asthma," Nutrients, vol. 5, no. 6, pp. 2128-2143, 2013.

[34] A. Funaro, X. Wu, M. Song et al., "Enhanced anti-inflammatory activities by the combination of luteolin and tangeretin," Journal of Food Science, vol. 81, no. 5, pp. H1320-H1327, 2016.

[35] C. Nunes, L. Almeida, R. M. Barbosa, and J. Laranjinha, "Luteolin suppresses the JAK/STAT pathway in a cellular model of intestinal inflammation," Food \& Function, vol. 8, no. 1, pp. 387-396, 2017.

[36] N. Aziz, M.-Y. Kim, and J. Y. Cho, "Anti-inflammatory effects of luteolin: a review of in vitro, in vivo, and in silico studies," Journal of Ethnopharmacology, vol. 225, pp. 342-358, 2018.

[37] J. Mlcek, T. Jurikova, S. Skrovankova, and J. Sochor, "Quercetin and its anti-allergic immune response," Molecules, vol. 21 , no. 5, p. 623, 2016.

[38] H. G. Ulusoy and N. Sanlier, "A minireview of quercetin: from its metabolism to possible mechanisms of its biological activities," Critical Reviews in Food Science and Nutrition, vol. 60, no. 19, pp. 3290-3303, 2020.

[39] M. R. Cesarone, G. Belcaro, S. Hu et al., "Supplementary prevention and management of asthma with quercetin phytosome: a pilot registry," Minerva Medica, vol. 110, no. 6, pp. 524-529, 2019.

[40] A. P. Rogerio, A. Kanashiro, C. Fontanari et al., "Anti-inflammatory activity of quercetin and isoquercitrin in experimental murine allergic asthma," Inflammation Research, vol. 56, no. 10, pp. 402-408, 2007.

[41] S. Zhu, H. Wang, J. Zhang et al., "Antiasthmatic activity of quercetin glycosides in neonatal asthmatic rats," 3 Biotech, vol. 9, no. 5, p. 189, 2019.

[42] E. A. Townsend and C. W. Emala Sr., "Quercetin acutely relaxes airway smooth muscle and potentiates $\beta$-agonist-induced relaxation via dual phosphodiesterase inhibition of PLC $\beta$ and PDE4," American Journal of Physiology. Lung Cellular and Molecular Physiology, vol. 305, no. 5, pp. L396L403, 2013.
[43] H.-I. Cho, J.-H. Park, H.-S. Choi et al., "Protective mechanisms of acacetin against D-galactosamine and lipopolysaccharide-induced fulminant hepatic failure in mice," Journal of Natural Products, vol. 77, no. 11, pp. 2497-2503, 2014.

[44] D. Wu, Y. Wang, H. Zhang, M. Du, and T. Li, "Acacetin attenuates mice endotoxin-induced acute lung injury via augmentation of heme oxygenase-1 activity," Inflammopharmacology, vol. 26, no. 2, pp. 635-643, 2018.

[45] W. C. Huang and C. J. Liou, "Dietary acacetin reduces airway hyperresponsiveness and eosinophil infiltration by modulating eotaxin-1 and th2 cytokines in a mouse model of asthma," Evidence-based Complementary and Alternative Medicine: eCAM, vol. 2012, Article ID 910520, 11 pages, 2012.

[46] M.-H. Pan, C.-S. Lai, Y.-J. Wang, and C.-T. Ho, "Acacetin suppressed LPS-induced up-expression of iNOS and COX-2 in murine macrophages and TPA-induced tumor promotion in mice," Biochemical Pharmacology, vol. 72, no. 10, pp. 1293-1303, 2006.

[47] S. Singh, P. Gupta, A. Meena, and S. Luqman, "Acacetin, a flavone with diverse therapeutic potential in cancer, inflammation, infections and other metabolic disorders," Food and Chemical Toxicology, vol. 145, Article ID 111708, 2020.

[48] M. Rincon and C. G. Irvin, "Role of IL-6 in asthma and other inflammatory pulmonary diseases," International Journal of Biological Sciences, vol. 8, no. 9, pp. 1281-1290, 2012.

[49] S. N. Harbour, D. F. DiToro, S. J. Witte et al., "T (H)17 cells require ongoing classic IL-6 receptor signaling to retain transcriptional and functional identity," Science Immunology, vol. 5, no. 49, 2020.

[50] M. T. Zdrenghea, H. Makrinioti, A. Muresan, S. L. Johnston, and L. A. Stanciu, "The role of macrophage IL-10/innate IFN interplay during virus-induced asthma," Reviews in Medical Virology, vol. 25, no. 1, pp. 33-49, 2015.

[51] M. Berry, C. Brightling, I. Pavord, and A. Wardlaw, "TNF- $\alpha$ in asthma," Current Opinion in Pharmacology, vol. 7, no. 3, pp. 279-282, 2007.

[52] Y. Sun, Z. Shi, B. Liu et al., "YKL-40 mediates airway remodeling in asthma via activating FAK and MAPK signaling pathway," Cell Cycle, vol. 19, no. 11, pp. 1378-1390, 2020.

[53] G. L. Johnson and R. Lapadat, "Mitogen-activated protein kinase pathways mediated by ERK, JNK, and p38 protein kinases," Science, vol. 298, no. 5600, pp. 1911-1912, 2002.

[54] M. Paplińska, J. Hermanowicz-Salamon, P. Nejman-Gryz et al., "Expression of eotaxins in the material from nasal brushing in asthma, allergic rhinitis and COPD patients," Cytokine, vol. 60, no. 2, pp. 393-399, 2012.

[55] Y.-H. Shieh, H.-M. Huang, C.-C. Wang, C.-C. Lee, C.-K. Fan, and Y.-L. Lee, "Zerumbone enhances the Th1 response and ameliorates ovalbumin-induced Th2 responses and airway inflammation in mice," International Immunopharmacology, vol. 24, no. 2, pp. 383-391, 2015.

[56] E. Mahsum, R. Nurjan, and Y. Abdiryim, "Effect of uighur medicine Hyssopus officinalis L. Ethly aectateon inflannnatory responsein asthma rats," Science \& Technology Review, vol. 29, no. 30, pp. 52-56, 2011.

[57] M. Hou, M. Zhu, X. Ma et al., "Effects of Uygur medicine Hyssopus officinalis L. on serum IL-17 level and balance of Th1/Th2 of asthma rats," China Journal of Modern Medicine, vol. 20, no. 3, pp. 365-368, 2010. 